\title{
Quantum Mechanical Studies of Physicochemical Properties on Estradiol and Isomer
}

\author{
Aparna Das ${ }^{1}$ and Bimal Banik ${ }^{1}$ \\ ${ }^{1}$ Prince Mohammad Bin Fahd University
}

May 11, 2020

\begin{abstract}
The current study investigates the correlation between biological activity and physicochemical properties of a few specific estradiol isomers. Theoretical studies on the physicochemical properties of estradiol isomers were performed using different quantum mechanical methods. The computational methods used in this study include the Density Functional Theory (DFT) method, the Hartree-Fock (HF) method and Semi-empirical (AM1) method. Some physicochemical properties such as dipole moment, molecular weight, the energy of the highest occupied molecular orbital (E HOMO), the energy of the lowest unoccupied molecular orbital (E LUMO), polarizability, the octanol-water partition coefficient (Log P), polar surface area (PSA) the number of hydrogen bond donors (HBDs) and the number of hydrogen bond acceptors (HBAs), the surface area, volume of the molecule, and ovality are calculated for the isomers. However, only dipole moment values are suitable to identify a correlation of experimental biological activity of estradiol isomers. To the best of our knowledge, this is the first report on the relationships between dipole moment and biological activities of estradiol isomers. It is observed that the active compound has a significantly higher dipole moment value compared to the inactive compound. We have also analyzed the geometrical and graphical models of these isomers and related compounds to evaluate the differences in the molecular charge distributions.
\end{abstract}

\section{Hosted file}

Das A full text.doc available at https://authorea.com/users/320464/articles/450002-quantummechanical-studies-of-physicochemical-properties-on-estradiol-and-isomer 